\title{
Exhaled Breath Condensate Nitrate Levels are Inversely Associated with the Body Mass Index of Patients without Respiratory Disease
}

Ana Fernandez-Bustamante ${ }^{1,2 *}$, Tamas Seres ${ }^{1}$, Amanda Agazio ${ }^{1,2}$, Alexander T. Pennington ${ }^{1,3}$, Uwe Christians ${ }^{1,3}$, Jelena Klawitter ${ }^{1,3}$ and John E. Repine ${ }^{2,4}$

${ }^{1}$ Department of Anesthesiology, University of Colorado School of Medicine, Aurora, CO, USA

${ }^{2}$ Webb-Waring Center, University of Colorado School of Medicine, Aurora, CO, USA

${ }^{3}$ iC42 Integrated Solutions in Systems Biology, University of Colorado School of Medicine, Aurora, CO, USA

${ }^{4}$ Department of Medicine, University of Colorado School of Medicine, Aurora, CO, USA

\begin{abstract}
Background: Repeated observations suggest that the incidence and/or severity of the Acute Respiratory Distress Syndrome (ARDS) is lower in mildly obese humans compared to lean subjects, phenomenon called the Obesity-ARDS Paradox. A reduced lung nitrosative stress could contribute to this unexplained protection. We measured levels of nitrate, the most oxidized nitric oxide (NO) metabolite, and other related metabolites in the exhaled breath condensate (EBC) of obese (composed of both overweight or mildly obese) and normal weight patients.
\end{abstract}

Methods: We studied patients without respiratory disease immediately after starting mechanical ventilation for elective surgery. We performed targeted metabolomics analyses of EBC and blood samples. We measured concentrations of arginine, asymmetrical dimethylarginine (ADMA), symmetrical dimethylarginine (SDMA), nitrite, and nitrate and then analyzed their relationship to body mass index (BMI). We compared patients classified as $\mathrm{BMI}<25$ (normal) or 25-34.9 (obese).

Results: 21 patients were included in the analysis: 5 with a BMI<25 and 16 with a BMI 25-34.9. Concentrations of nitrate in EBC, but not in plasma, inversely correlated with BMI. EBC nitrate levels positively correlated with EBC nitrite but not with plasma nitrate levels. EBC nitrite levels inversely correlated with plasma nitrite levels. Patients with a BMI 25-34.9 had significantly lower EBC nitrate levels than patients with a BMI $<25$.

Conclusion: Our results suggest a lower nitrosative stress in the lungs of overweight and mildly obese patients compared to normal weight patients. This observation deserves further evaluation as a possible contributing factor to the Obesity ARDS Paradox.

Keywords: Nitrate; Exhaled breath condensate; Nitric oxide pathway; Body mass index; Obesity-ARDS paradox

\section{Introduction}

Obesity is generally associated with an increased incidence of medical problems and overall mortality [1-3]. However, several studies suggest better than expected outcomes associated with mild obesity, a phenomenon termed the "obesity paradox" [3-10]. The obesity paradox usually refers to the unexplained decreased overall mortality and decreased mortality from cardiovascular events in otherwise healthy mildly obese patients compared to normal weight patients $[7,10,11]$. Repeated studies report a decreased incidence and/or reduced mortality from the acute respiratory distress syndrome (ARDS) [1215 ] in obese patients compared to normal weight individuals, although a few conflicting findings exist $[15,16]$. In addition, obesity has been associated with a delayed ARDS onset during an ICU stay [15] and lower plasma inflammatory markers during ARDS development [17]. We have labeled this unexplained phenomenon as the "obesityARDS paradox" $[18,19]$. These observations are intriguing even though many of these studies leading to this paradox suffer from their retrospective nature, exclusion of morbidly obese patients, the overall low incidence of ARDS and the lack of accurate information about possible respiratory, metabolic and cardiovascular comorbidities often associated with obesity.

The absence of any known explanatory mechanisms triggers skepticism about the obesity-ARDS paradox. Nonetheless, the repeated observations suggesting that mild obesity may protect against ARDS deserve considerable interest due to the absence of successful therapies and the unchanged high fatality of the ARDS. Thus, an increased understanding of any possible endogenous mechanisms that "naturally" down-modulate ARDS could provide some novel insights into identifying promising pathways for ARDS prevention in nonobese individuals.
Obesity impairs the nitric oxide (NO) metabolism. Obese patients have increased plasma levels of asymmetric dimethylarginine (ADMA) [20], a natural inhibitor of the endothelial nitric oxide synthase (NOS) function and nitric oxide production [20,21]. Endothelial NOS dysfunction decreases NO-dependent vasodilation and contributes to hypertension and other cardiovascular complications often seen with obesity [20]. Recent studies focused on obesity-related asthma in adults suggest that obese adult asthmatics have a reduced fractional NO concentration in exhaled air (FeNO) related to an impairment in the arginine/ADMA pathway [22,23]. Moreover, FeNO increases in morbidly obese patients without asthma after weight loss following bariatric surgery [24]. The effect of obesity on exhaled NO levels in mildly obese humans without diagnosed asthma is still unclear $[25,26]$. This effect may be significant since NO is involved in the pulmonary immune defense and repair $[27,28]$ and $\mathrm{NO}$ is increased in the bronchoalveolar lavage (BAL) fluid and lungs of patients during ARDS development [29].

*Corresponding author: Ana Fernandez-Bustamante, MD, PhD, Department of Anesthesiology and Webb-Waring Center, University of Colorado SOM $12631 \mathrm{E}$ 17th Ave AO-1, R2012, MS 8202 Aurora, CO, 80045, USA, Tel: +001-303-7242935; E-mail: Ana.Fernandez-Bustamante@ucdenver.edu

Received November 01, 2014; Accepted February 24, 2015; Published February 28, 2015

Citation: Fernandez-Bustamante A, Seres T, Agazio A, Pennington AT, Christians U, et al. (2015) Exhaled Breath Condensate Nitrate Levels are Inversely Associated with the Body Mass Index of Patients without Respiratory Disease. J Pulm Respir Med 5: 243. doi:10.4172/2161-105X.1000243

Copyright: (C) 2015 Fernandez-Bustamante A, et al. This is an open-access article distributed under the terms of the Creative Commons Attribution License, which permits unrestricted use, distribution, and reproduction in any medium, provided the original author and source are credited. 
Citation: Fernandez-Bustamante A, Seres T, Agazio A, Pennington AT, Christians U, et al. (2015) Exhaled Breath Condensate Nitrate Levels are Inversely Associated with the Body Mass Index of Patients without Respiratory Disease. J Pulm Respir Med 5: 243. doi: 10.4172/2161-105X.1000243

Page 2 of 8

In exhaled breath condensate (EBC), $\mathrm{NO}$ is usually measured as a combination of its water-soluble metabolites nitrite and nitrate, where nitrate represents the most oxidized NO metabolite that is not reducible by human cells [30]. Therefore, a dampened pulmonary nitrate concentration and nitrosative stress could confer some resistance to ARDS development and theoretically contribute to the obesity ARDS paradox.

We hypothesized that obese adults without any respiratory disease would have lower concentrations of nitrate in their EBC than normal weight patients. In previously collected samples of EBC and plasma [31], we analyzed and compared nitric oxide and their related pathway metabolites using targeted metabolomics analyses.

\section{Materials and Methods}

The experimental protocol was approved by the University of Colorado Multiple Institutional Review Board (Aurora, Colorado, US) before conducting the study. All patients signed and were given their informed written consent and the study was carried out following all applicable good clinical practice guidelines.

\section{Patients and study protocol}

We performed analyses of previously collected samples of exhaled breath condensate (EBC) and plasma obtained from surgical patients without any respiratory disease immediately after the start of mechanical ventilation [31]. These surgical patients had been enrolled in the previously published study on lung injury biomarkers during mechanical ventilation [31]. Briefly, study patients were subjected to general anesthesia for elective knee replacement and prospectively randomized to receive volume controlled ventilation with a tidal volume of 6 or $10 \mathrm{~mL} / \mathrm{kg}$ predicted body weight (PBW) [32] during mechanical ventilation. Exclusion criteria included: American Society of Anesthesiologists (ASA) class 4; age $\geq 70$ years; emergency procedure; status post pneumonectomy; diagnosed chronic obstructive pulmonary disease (COPD), emphysema, asthma, pulmonary hypertension, sleep apnea or any other respiratory disease; oxygen-therapy during last month; tobacco use in the last 5 years; Body Mass Index $(\mathrm{BMI}) \geq 35 \mathrm{~kg} / \mathrm{m}^{2}$; immunosupression within 3 months prior to the procedure; diagnosed infection; or shock. Patients were randomized to receive a tidal volume $\left(\mathrm{V}_{\mathrm{T}}\right)$ of either $6\left(\mathrm{~V}_{\mathrm{T}} 6\right)$ or 10 $\left(\mathrm{V}_{\mathrm{T}} 10\right) \mathrm{mL} / \mathrm{kg}$ predicted body weight (PBW) [32,33], the respiratory rate was titrated for eucapnia (end-tidal $\mathrm{CO}_{2}$ partial pressure, $\mathrm{E}_{\mathrm{T}} \mathrm{CO}_{2}$, $30-40 \mathrm{mmHg}$ ), and the same following ventilatory settings were used in all patients: inspiratory: expiratory (I:E) ratio 1:2, inspiratory pause $5 \%$, inspiratory oxygen fraction $\left(\mathrm{FiO}_{2}\right) 0.5$, positive end-expiratory pressure (PEEP) $5 \mathrm{cmH}_{2} \mathrm{O}$. Anesthetic management was otherwise similar in all patients. Immediately after starting mechanical ventilation $(0 \mathrm{~min}$ time point), before the surgical incision, we initiated the collection of $\mathrm{EBC}$ and blood samples. EBC samples were collected with an Rtube ${ }^{\mathrm{TM}}$ breath condensate vial (Respiratory Research, Inc., Austin, TX) placed in the expiratory limb of the ventilatory circuit for 20 minutes. The heat moisture exchange filter adjacent to the $\mathrm{Y}$ connector was removed and no humidification was added to the ventilatory circuit. 20 minutes later, EBC samples were immediately placed in regular ice, volume measured and aliquoted into vials pre-washed with deionized water and frozen at $-80 \mathrm{C}$ until analysis. Venous blood samples were collected in EDTA vials and immediately transported in regular ice to the laboratory. Samples were centrifuged at 2,000 rpm for 10 minutes, plasma volume aliquoted and frozen at $-80^{\circ} \mathrm{C}$ until analysis.

Samples of EBC and blood were repeated after 60 minutes in the original study [31], but for the current study on NO-related measurements only 0 minute time point samples were used to avoid 60 minute of mechanical ventilation as a potential confounder. For the current study we only included patients with enough remaining volume of EBC and plasma samples for the proposed measurements. Samples from 0minute time points from both ventilatory groups were pooled together for this study because no differences in metabolites concentrations were observed in the original study.

\section{Demographics and physiology}

We recorded age, gender, ASA classification, height, weight and medical conditions for all patients. The Body Mass Index (BMI) was calculated for all patients as BMI=weight $(\mathrm{kg}) \times 1 /$ height $(\mathrm{m})^{2}$. BMI was used to classify the degree of obesity of patients based on accepted limits [33], as follows: normal weight (BMI 18.5-24.9) and obese (BMI 25-34.9). As mentioned above, BMI $\geq 35$ was an exclusion criterion in our original study[31]. Physiology parameters including: mean blood pressure, heart rate, temperature, peripheral saturation of oxygen by pulse-oximetry $\left(\mathrm{SpO}_{2}\right)$, end-tidal partial pressure of carbon dioxide $\left(\mathrm{E}_{\mathrm{T}} \mathrm{CO}_{2}\right)$, minute volume ventilation, and peak and plateau airway pressures were recorded during EBC sample collections.

\section{Nitric oxide metabolites and related endothelial dysfunction biomarkers}

Concentrations of NO metabolites nitrite and nitrate in EBC and plasma were independently measured using a dedicated HPLC system (ENO-20, Eicom, San Diego, CA) [34] and reported techniques as described in detail in the original study [31]. Concentrations of arginine, ADMA and SDMA were additionally measured by HPLCtandem mass spectrometry (LC-MS/MS). In brief: the API5500 mass spectrometer for EBC and API4000 mass spectrometer for plasma (both $\mathrm{AB}$ Sciex, Concord, $\mathrm{ON}$ ) were run in the positive electrospray ionization mode (ESI) using multiple reaction monitoring (MRM). The following ion transitions were used (mass/charge, $\mathrm{m} / \mathrm{z}=$ ): arginine: $\mathrm{m} /$ $\mathrm{z}=175.2 \rightarrow 70.1$; ADMA: $203.2 \rightarrow 46.2$; SDMA: $203.2 \rightarrow 172.2$; d7-ADMA (internal standard): $210.2 \rightarrow 77.2$. Briefly, to $100 \mu \mathrm{L}$ of plasma or 200 $\mu \mathrm{L}$ of EBC, $50 \mu \mathrm{L}$ of internal standard containing solution $(50 \mu \mathrm{M} \mathrm{d} 7-$ ADMA in HPLC-grade water) and $40 \mu \mathrm{L}$ of $500 \mathrm{mM}$ DTT solution were added. For protein precipitation, $400 \mu \mathrm{L}$ (for plasma) or $200 \mu \mathrm{L}$ (for EBC) of $0.05 \%$ trifluoric acid plus $0.1 \%$ formic acid containing acetonitrile solution were added to the sample. The sample was finally vortexed for 5 minutes, centrifuged for 10 minutes at 13,000g and transferred into an HPLC vial. $20 \mu \mathrm{L}$ (for plasma) or $100 \mu \mathrm{L}$ (for $\mathrm{EBC}$ ) of the supernatant were injected onto a $4.6 \times 12.5 \mathrm{~mm}$ guard/ extraction column (Eclipse XDB-C8, $5 \mu \mathrm{m}$, Agilent Technologies, Palo Alto, CA) inline with a $3.0 \times 150 \mathrm{~mm}$ analytical column (RP-Amide, $3.5 \mu \mathrm{m}$, Supelco, St. Louis, MO). The gradient started at 3\% methanol and $97 \% 10 \mathrm{mM}$ ammonium formate buffer and was maintained at a flow of $0.8 \mathrm{~mL} / \mathrm{min}$ throughout the assay. At minute 4.5 , the solvent gradient reached 25\% methanol; after this the methanol content was raised to $98 \%$ and held for an additional $1.5 \mathrm{~min}$. Hereafter the column was re-equilibrated to the starting conditions for 2 minutes. Figure 1 summarizes the key metabolic pathways and the relationships between the measured metabolites.

\section{Statistical analysis}

All variables were graphically depicted and summarized using means and standard deviations if normally distributed or median and interquartile range (IQR) if not normally distributed. Not normally distributed metabolite concentrations were logarithmically transformed for analyses. The statistical association between BMI and the log-transformed metabolite concentrations in EBC, plasma and 
Citation: Fernandez-Bustamante A, Seres T, Agazio A, Pennington AT, Christians U, et al. (2015) Exhaled Breath Condensate Nitrate Levels are Inversely Associated with the Body Mass Index of Patients without Respiratory Disease. J Pulm Respir Med 5: 243. doi: 10.4172/2161-105X.1000243

Page 3 of 8

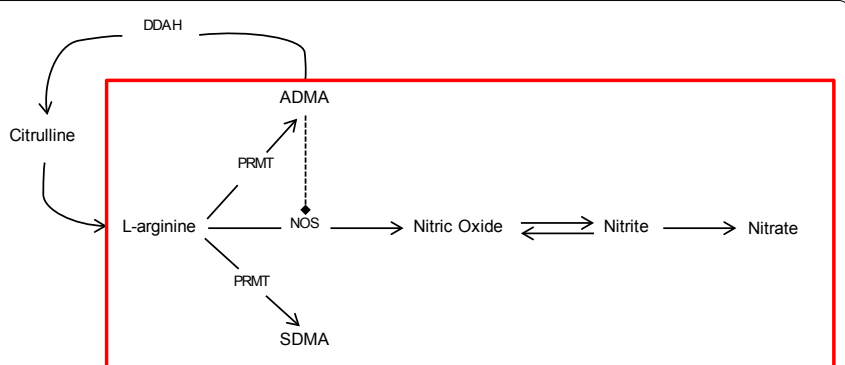

Figure 1: Nitric oxide pathway. Pathway relationships of studied metabolites. The primary focus of this study is included in the red rectangle. (ADMA: Asymmetrical Dimethylarginine; DDAH: Dimethylarginine Dimethylaminohydrolase; NOS: Nitric Oxide Synthase; PRMT: Protein Arginine Residues Methyl-Transferases; SDMA: Symmetrical Dimethylarginine).

$\mathrm{EBC} /$ plasma ratios, and between the metabolites themselves, were analyzed by Pearson correlation. Patients were classified based on the following commonly used BMI ranges: $\mathrm{BMI}<25$ (normal weight), BMI 25-34.5 (obese). EBC and plasma concentrations as well as and EBC/ plasma ratios of measured metabolites were statistically compared within the BMI groups with ANOVA and Bonferroni post-hoc test for pair comparisons. All statistical analyses were two-tailed and performed with SPSS version 21 (IBM, New York, NY). Significance was accepted at $\mathrm{p}<0.05$.

\section{Results}

\section{Demographics and physiology}

A total of 21 patients were included in this study. To be included in the analysis, remaining EBC and blood samples had to have sufficient volume for the present additional measurements. 5 patients had a $\mathrm{BMI}<25,16$ patients had a BMI 25-34.9. Per study inclusion and exclusion criteria, no patients had any respiratory disease, respiratory pharmacological treatments or smoking habits. In addition, no significant differences were found between the two BMI groups in terms of age, gender, height and ASA classification (Table 1). Despite having a comparable height, patients in the BMI 25-34.5 group had significantly greater weight than patients in the $\mathrm{BMI}<25$ group. The pre-existing diagnosis of hypertension was significantly more common in the BMI 25-34.9 group. Hemodynamic parameters (blood pressure, heart rate), temperature, oxygenation by pulse oximetry, end-tidal partial pressure of $\mathrm{CO}_{2}$, minute volume ventilation, peak and plateau airway pressures were comparable between the groups.

\section{Concentrations of metabolites of the nitric oxide pathway in EBC and plasma samples}

Table 2 shows the concentrations in EBC and plasma samples of all patients combined as well as in patients classified in both BMI groups. Measurements were not normally distributed and were transformed logarithmically for statistical analysis. EBC nitrate levels in BMI 25-34.9 patients were significantly lower than in the $\mathrm{BMI}<25$ patients. Plasma concentrations of nitrate or other metabolites were not significantly different between both BMI groups. The EBC/plasma ratios for all metabolites are also shown in Table 2 for both BMI groups.

The BMI had a significant inverse association with the logarithmically transformed EBC nitrate concentrations and the EBC/ plasma nitrate ratios, but not with plasma nitrate concentrations (Figure 2). BMI did not correlate with any other metabolites in $\mathrm{EBC}$ or plasma samples, or with any EBC/plasma ratios.

Concentrations of $\mathrm{EBC}$ nitrate showed a positive association with EBC nitrite levels (Figure 3) but were not correlated with the concentrations of plasma nitrate, EBC arginine or EBC ADMA levels. Concentrations of EBC nitrite showed an inverse association with plasma nitrite levels. EBC arginine or ADMA levels were not associated with their corresponding plasma concentrations.

\section{Distribution of EBC/plasma metabolites based on BMI}

Figure 4 depicts the distribution of EBC and plasma concentrations of nitrate and other individual metabolites in both BMI groups. EBC nitrate concentrations in the BMI 25-34.9 were significantly lower than in the $\mathrm{BMI}<25$ group. The concentrations of $\mathrm{EBC}$ nitrite showed a trend to be lower in the BMI 25-34.9 group than in BMI $<25$ group, but it did not reach statistical significance. Plasma nitrate and nitrite concentrations were similar in both BMI groups. The sum concentrations of nitrite and nitrate, traditionally labeled as NOx, showed the same trend as nitrite but did not reach statistical significance. No significant differences were observed in other studied metabolites in EBC or plasma samples.

\section{Discussion}

Our findings suggest that the BMI inversely correlates with nitrate concentrations in the exhaled breath condensate (EBC) of surgical patients without any respiratory disease. Our obese patients (BMI 25-34.9) had significantly lower EBC nitrate levels than patients with normal weights $(\mathrm{BMI}<25)$.

In our study, increasing BMI was significantly and inversely correlated with the concentration of nitrate in EBC samples. Obese patients had significantly lower EBC nitrate levels and a trend toward lower EBC nitrite levels compared to normal weight patients. Nitrate constitutes the most oxidized NO metabolite (Figure 1) and while nitrite can be reduced to NO by different cell types, human cells cannot reduce nitrate to nitrite [31]. Both nitrite and nitrate have been measured before in EBC samples from spontaneously breathing patients, and our results are similar to reported levels from healthy adults [35-37]. The decreased NO production and nitrosative stress in EBC of obese patients without respiratory disease has not been previously reported. In a study by Holguin, et al. [26] BMI was directly associated with BAL leptin levels but not with the BAL NO products in humans with and without asthma. The difference between these findings and our findings may reflect the source of the samples analyzed (BAL instead of $\mathrm{EBC})$ and/or the analytical technique for measuring nitrite and nitrate (the Griess reaction versus the more accurate HPLC approach used in the present investigation).

Nitric oxide production, nitrosative stress and protein nitration increases in both human ARDS and animal models of ARDS [29,3842]. Although the BAL levels of nitrite and nitrate did not predict ARDS development in critically ill humans at risk for ARDS, high BAL levels in ARDS patients were associated with worse outcomes and increased mortality [29]. Moreover, recent findings in septic patients point to plasma NOS uncoupling as a feature of ARDS development but the responsible mechanisms remain unclear [43]. NO attenuation could be a protective mechanism against ARDS development. In a sheep model of burn and inhalation-induced ARDS, IV administration of an iNOS inhibitor decreased plasma levels of NO and attenuated acute lung injury features (lung edema, compliance, gas exchange) [40] . Nevertheless, it is not known if reduced NO or NO metabolites (nitrite and nitrate) protect against ARDS in humans.

Why is there interest in the lower nitrosative stress in the lung of obese patients? Severe obesity is widely accepted as a risk factor for increased mortality and morbidity [5,11], but being overweight and having mild obesity may have a paradoxical effect in lowering 
Citation: Fernandez-Bustamante A, Seres T, Agazio A, Pennington AT, Christians U, et al. (2015) Exhaled Breath Condensate Nitrate Levels are Inversely Associated with the Body Mass Index of Patients without Respiratory Disease. J Pulm Respir Med 5: 243. doi: $10.4172 / 2161-105 X .1000243$

Page 4 of 8

\begin{tabular}{|c|c|c|c|}
\hline & All & $\mathrm{BMI}<25$ & BMI 25-34.9 \\
\hline \multicolumn{4}{|l|}{ Number } \\
\hline Age (years) & 21 & 5 & 16 \\
\hline Gender distribution & $63.3 \pm 5.3$ & $62.2 \pm 7.0$ & $63.7 \pm 5.0$ \\
\hline Male & $7(33.3 \%)$ & $1(20.0 \%)$ & $6(37.5 \%)$ \\
\hline Female & $14(66.7 \%)$ & $4(80.0 \%)$ & $10(62.5 \%)$ \\
\hline Height (cm) & $168.7 \pm 9.8$ & $167.4 \pm 15.4$ & $169.1 \pm 7.9$ \\
\hline Weight (kg) & $78.9 \pm 13.7$ & $66.5 \pm 15.0$ & $82.8 \pm 11.1^{*}$ \\
\hline \multicolumn{4}{|l|}{ ASA classification } \\
\hline 1 & $1(4.8 \%)$ & $1(20.0 \%)$ & $0(0.0 \%)$ \\
\hline 2 & $15(71.4 \%)$ & $4(80.0 \%)$ & $11(68.8 \%)$ \\
\hline 3 & $5(23.8 \%)$ & $0(0.0 \%)$ & $5(31.3 \%)$ \\
\hline \multicolumn{4}{|l|}{ Comorbidities } \\
\hline Hypertension & $9(42.9 \%)$ & $0(0.0 \%)$ & $9(56.3 \%)^{*}$ \\
\hline Gastroesophageal reflux disease & $4(19.0 \%)$ & $0(0.0 \%)$ & $4(25.0 \%)$ \\
\hline Diabetes Mellitus & $4(19.0 \%)$ & $0(0.0 \%)$ & $4(25.0 \%)$ \\
\hline \multicolumn{4}{|l|}{ Physiology } \\
\hline Mean Blood Pressure $(\mathrm{mmHg})$ & $80.6 \pm 12.1$ & $73.0 \pm 9.7$ & $83.0 \pm 12.0$ \\
\hline Heart rate (beats/min) & $71.8 \pm 11.9$ & $76.0 \pm 11.8$ & $70.5 \pm 12.0$ \\
\hline Temperature (C) & $35.8 \pm 0.5$ & $35.6 \pm 0.4$ & $35.8 \pm 0.5$ \\
\hline $\mathrm{SpO}_{2}(\%)$ & $98.3 \pm 1.6$ & $98.8 \pm 1.3$ & $98.2 \pm 1.7$ \\
\hline $\mathrm{E}_{\mathrm{T}} \mathrm{CO}_{2}$ & $34.1 \pm 3.2$ & $33.2 \pm 5.3$ & $34.4 \pm 2.5$ \\
\hline Minute volume ventilation (L/min) & $5.1 \pm 0.8$ & $5.0 \pm 0.9$ & $5.1 \pm 0.8$ \\
\hline Peak Airway Pressure $\left(\mathrm{cmH}_{2} \mathrm{O}\right)$ & $18.0 \pm 3.1$ & $17.6 \pm 2.2$ & $18.2 \pm 3.4$ \\
\hline Plateau Airway Pressure $\left(\mathrm{cmH}_{2} \mathrm{O}\right)$ & $16.0 \pm 2.8$ & $15.4 \pm 1.5$ & $16.1 \pm 3.2$ \\
\hline
\end{tabular}

(ASA: American Society of Anesthesiologists Classification; BMI: Body Mass Index; $\mathrm{SpO}_{2}$ : Peripheral Saturation of Oxygen; $\mathrm{E}_{\mathrm{T}} \mathrm{CO}_{2}$ : End-tidal Carbon Dioxide Partial Pressure)

Table 1: Demographic and physiology details of study patients. The weight of patients in the BMI 25-34.9 group was significantly higher than in the BMl<25 group. Height was not significantly different between both groups. Patients in the BMI 25-34.9 group had significantly greater incidence of pre-existing diagnosis of hypertension than those in the $\mathrm{BMI}<25$ group. No other significant statistical differences were observed between groups. Data are expressed as Mean \pm SD or $\mathrm{n}(\%)$ where appropriate $\left({ }^{*}\right.$ $\mathrm{p}<0.05$ compared to $\mathrm{BMI}<25)$.

\begin{tabular}{|c|c|c|c|}
\hline & All patients & $\mathrm{BMI}<25$ & BMI 25-34.9 \\
\hline Number & 21 & 5 & 16 \\
\hline \multicolumn{4}{|l|}{ EBC } \\
\hline Arginine (nM) & $25.80(21.00)$ & $19.40(20.00)$ & $25.85(24.07)$ \\
\hline ADMA (nM) & $1.33(1.43)$ & $2.20\left(0^{\mathrm{a}}\right)$ & $1.17(1.27)$ \\
\hline SDMA (nM) & $1.69(0.86)$ & $1.70(0.30)$ & $1.68(0.29)$ \\
\hline Nitrite (mcM) & $0.20(0.96)$ & $1.11(1.05)$ & $0.15(0.58)$ \\
\hline Nitrate (mcM) & $0.62(1.09)$ & $1.54(1.48)$ & $0.54(0.53)^{\star}$ \\
\hline \multicolumn{4}{|l|}{ Plasma } \\
\hline Arginine (mcM) & $110.10(21.60)$ & $115.05(11.53)$ & $104.05(27.70)$ \\
\hline ADMA (mcM) & $0.68(0.13)$ & $0.59(0.22)$ & $0.70(0.12)$ \\
\hline SDMA (mcM) & $0.88(0.38)$ & $0.75(0.29)$ & $0.91(0.39)$ \\
\hline Nitrite (mcM) & $0.45(0.48)$ & $0.43(0.15)$ & $0.61(0.67)$ \\
\hline Nitrate (mcM) & $23.30(26.04)$ & $24.92(27.80)$ & $22.99(21.81)$ \\
\hline \multicolumn{4}{|l|}{ EBC/Plasma } \\
\hline Arginine $\left(\times 10^{-3}\right)$ & $0.26(0.16)$ & $0.22(0.20)$ & $0.27(0.21)$ \\
\hline ADMA $\left(\times 10^{-3}\right)$ & $1.77(1.61)$ & $2.82\left(0^{\mathrm{a}}\right)$ & $1.52(1.62)$ \\
\hline SDMA $\left(\times 10^{-3}\right)$ & $1.95(0.95)$ & $2.33(0.85)$ & $1.86(0.83)$ \\
\hline Nitrite $(\mathrm{mcM} / \mathrm{mcM})$ & $0.22(2.77)$ & $2.07(3.05)$ & $0.15(2.60)$ \\
\hline Nitrate $(\mathrm{mcM} / \mathrm{mcM})$ & $0.03(0.03)$ & $0.04(0.07)$ & $0.02(0.02)$ \\
\hline
\end{tabular}

(aOnly 1 EBC sample with a detectable measurement)

Table 2. Concentrations of measured metabolites in the EBC and plasma samples of study patients. Values represent median (interquartile range). Logarithmically transformed values were used for statistical analysis by ANOVA with Bonferroni post-hoc tests for paired comparisons. ( ${ }^{*} \mathrm{p}<0.05$ compared to BMI 25 ).

observed overall mortality, and even in decreasing mortality after certain cardiovascular events, a phenomenon known as the obesity paradox [5,9-11,44]. In addition, obese patients have better than expected surgical outcomes when critically ill $[45,46]$ and during the perioperative period $[8,47]$. Similarly, either a lower incidence and/or mortality from ARDS was observed in several studies of obese patients compared to normal weight patients [12-14]. Other studies have found a similar or increased incidence of ARDS with increasing BMI 
Citation: Fernandez-Bustamante A, Seres T, Agazio A, Pennington AT, Christians U, et al. (2015) Exhaled Breath Condensate Nitrate Levels are Inversely Associated with the Body Mass Index of Patients without Respiratory Disease. J Pulm Respir Med 5: 243. doi: $10.4172 / 2161-105 X .1000243$

A

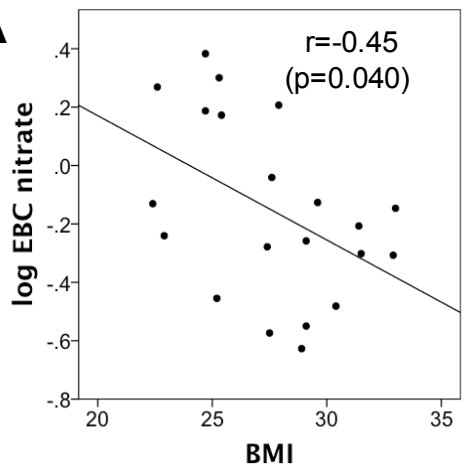

B
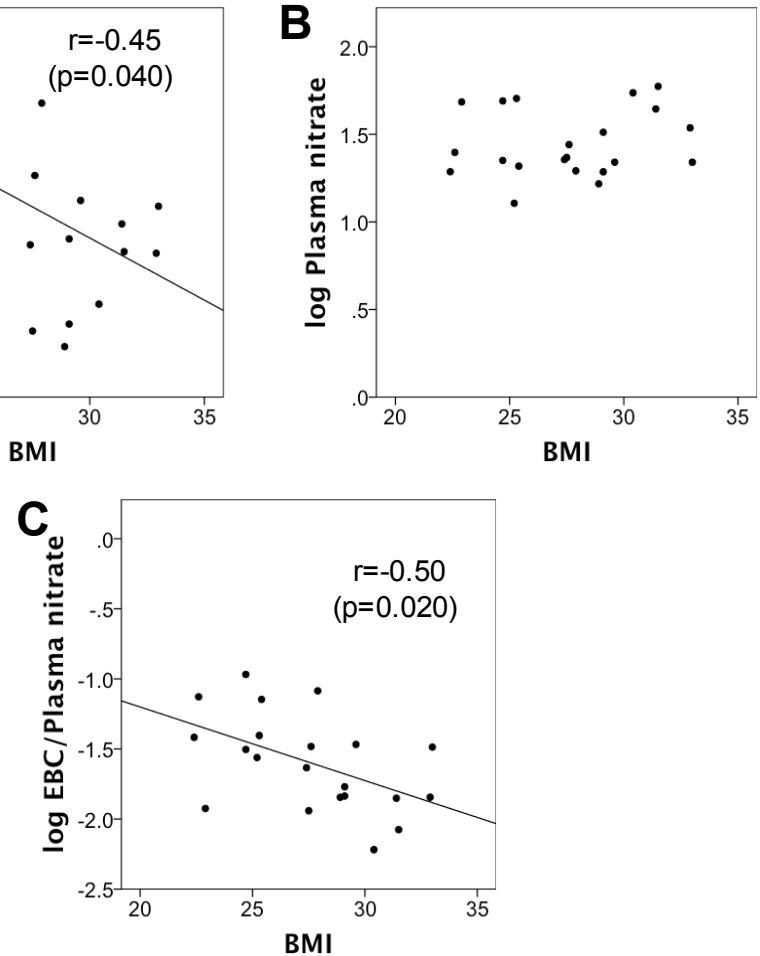

Figure 2: Correlations of BMI with nitrate concentrations. BMI showed a significant and inverse logarithmic association with $E B C$ nitrate $(A)$, but not with plasma nitrate (B). $\mathrm{EBC} /$ plasma nitrate ratio also inversely correlated with the BMI (C). The BMI did not correlate with other metabolites in EBC or plasma samples. $r$ represents the Pearson correlation coefficient.

A

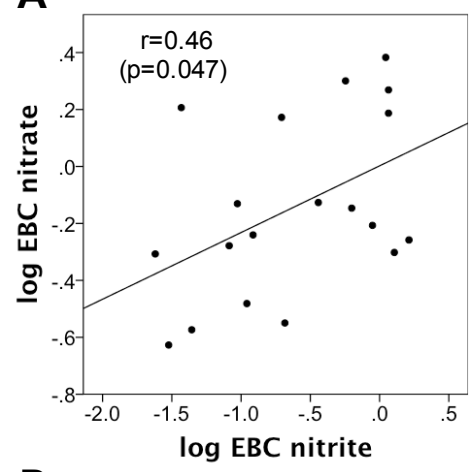

D

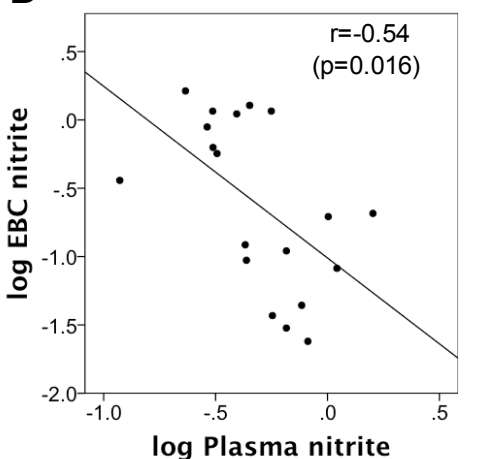

B

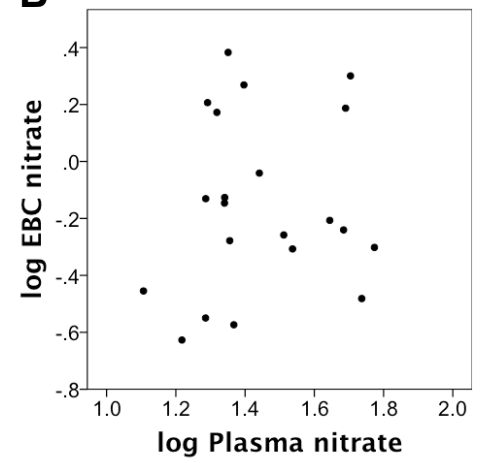

$\mathbf{E}$

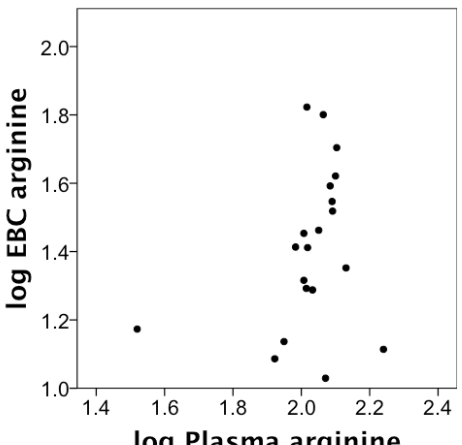

C

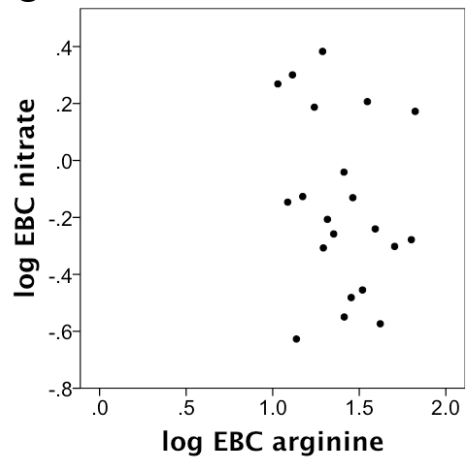

Figure 3: Correlation of EBC nitrate with other pathway targeted metabolites. EBC nitrate showed a positive association with EBC nitrite levels (A) but not with plasma nitrate levels (B) or precursor arginine levels in the EBC samples (C). Interestingly, EBC nitrite concentrations were inversely associated with plasma nitrite levels (D). EBC arginine levels did not show any association with plasma arginine concentrations (E). All statistical correlations were performed with logarithmically transformed values. $r$ represents the Pearson correlation coefficient. 
Citation: Fernandez-Bustamante A, Seres T, Agazio A, Pennington AT, Christians U, et al. (2015) Exhaled Breath Condensate Nitrate Levels are Inversely Associated with the Body Mass Index of Patients without Respiratory Disease. J Pulm Respir Med 5: 243. doi: $10.4172 / 2161-105 X .1000243$

Page 6 of 8
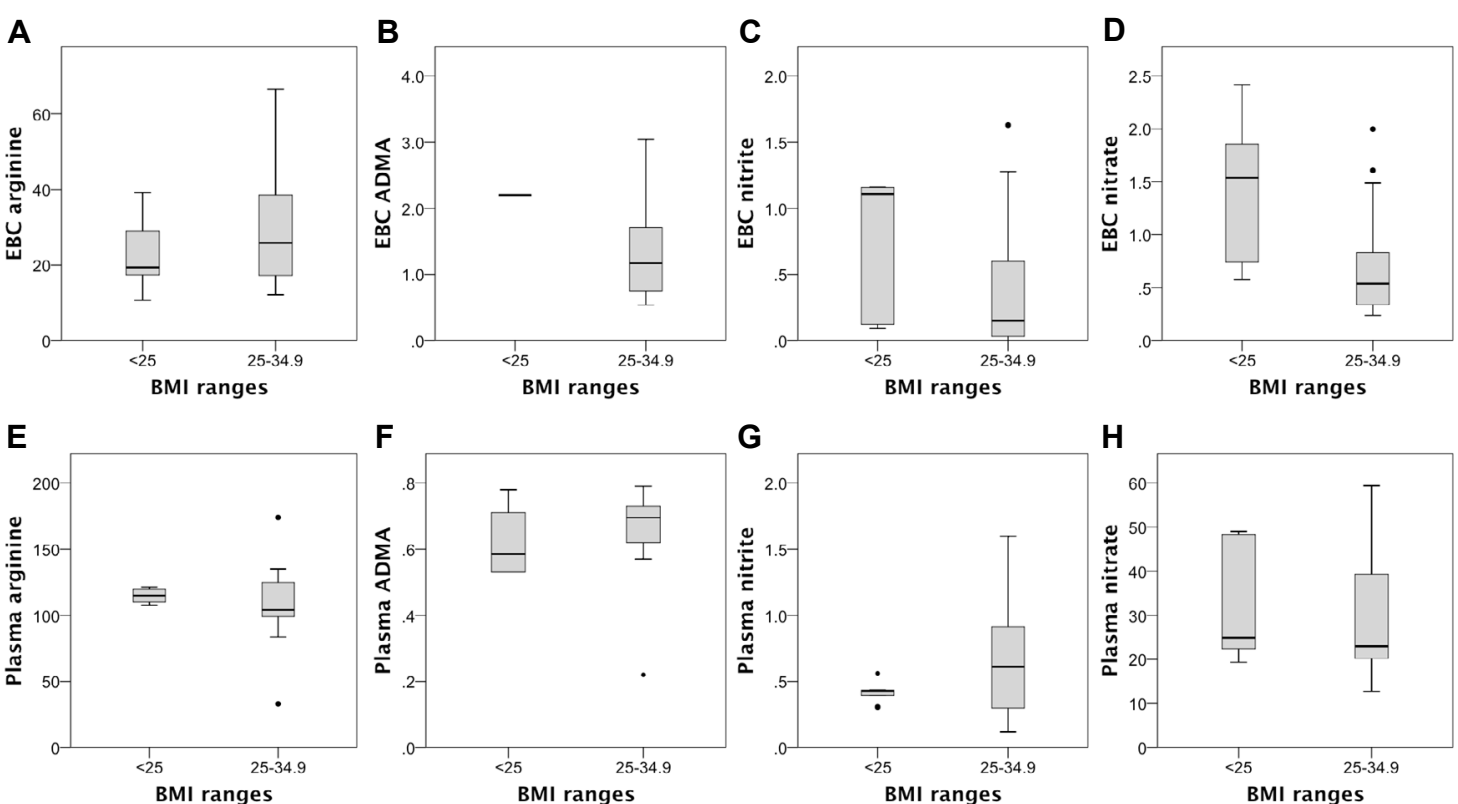

Figure 4: Distribution of concentrations of arginine, ADMA, nitrite and nitrate in exhaled breath condensate (EBC) and plasma samples of patients classified based on their Body Mass Index (BMI).

Graphs represent the distribution of concentrations of arginine, ADMA, nitrite and nitrate in EBC (A-D) and plasma (E-H) samples from patients in both BMI ranges. The $\mathrm{EBC}$ nitrate concentrations (D) were significantly lower in the BMI 25-34.9 patients than in the BMI<25 group. There was a trend to lower EBC nitrite concentrations (C) in the BMI 25-34.9 group, but it did not reach statistical significance. No significant differences were observed in other studied metabolites in EBC or plasma samples. Bars represent minimum, first quartile, median, third quartile, and maximum values. Outlier values are represented by full dots outside of bars. The logarithmically transformed values were used for statistical analysis, statistical significance accepted as $p<0.050$

that is not matched with a greater mortality $[15,16]$. As mentioned, this unexplained finding has been called the obesity-ARDS paradox $[18,19]$. Because of the increased obesity-associated comorbidities (sleep apnea, asthma, among others) and multiple other lifestyle confounders, conflicting findings or the mechanisms by which obesity affects outcomes are difficult to explain. The controversy nonetheless highlights an unanswered question. The current research trend is to investigate the contribution of cardiovascular fitness, glucose and lipid metabolic parameters and the effect of adiposity on the immune responses to explain this paradoxical protection regarding why certain mildly obese humans seem to resist ARDS $[5,17,48,49]$. Understanding the mechanisms of disease protection, be it dependent on adiposity or something else, would help explain this paradox and perhaps suggest new approaches for ARDS patients.

Measurements of arginine, ADMA and SDMA in EBC and plasma samples have also been previously reported in the literature [50-52]. Levels of ADMA are elevated in the EBC of children with asthma [52]. ADMA and the ADMA/arginine balance is also being explored in respiratory diseases associated with chronic hypoxia (chronic obstructive pulmonary disease, pulmonary fibrosis) [53,54] because of its well-known role as endogenous inhibitor of the nitric oxide synthase. Increases of ADMA leads to decreased endothelial NO availability and the altered regulation of the vascular tone, being one of the proposed mechanisms linking hypoxia with impaired vasodilation. The lung is a source of ADMA, and its concentration is regulated most significantly by its conversion to $\mathrm{NO}$ via the dimethylarginine dimethylaminohydrolase (DDAH) (Figure 1) [53]. It is difficult to compare our ADMA concentrations in EBC to those observed by Carraro et al. [52] in healthy children, where EBC ADMA levels were normalized to tyrosine concentrations, not available in our study. Our measured concentrations of ADMA, SDMA and arginine in EBC samples are similar to those reported in healthy adolescents [51] and adults [55]. We did not observe significant differences in BMI-classified patients on concentrations of ADMA, arginine/ADMA or nitrate/ ADMA ratios in EBC or plasma samples, but these may be specific findings to obesity-related asthma [22].

The positive associations observed between EBC nitrite concentrations with EBC nitrate and inverse with EBC ADMA levels reinforce the reliability of our measurements and fits with the known pathway relationships described in Figure 1. The lack of association between EBC nitrate and plasma nitrate levels suggests some degree of independence of the nitrosative stress in the lung respective to the systemic circulation. Although obesity is known to be associated with increased plasma levels of ADMA, we did not observe significant differences between our normal weight and mildly obese patients. ADMA impairs endothelial NOS function and contributes to the cardiovascular complications often seen with obesity [20]. Moreover, recent findings suggest that eNOS impairment may also contribute to obesity by attenuating or preventing increases in metabolic activity in the adipose tissue [56]. Ware, et al. [43] recently found significantly lower citrulline plasma levels in ARDS compared to non-ARDS patients. These authors suggested that a reduced precursor supply could lead to NOS uncoupling resulting in greater formation of peroxynitrites and oxidative injury. In our study we did not study peroxynitrite or nitrosylated proteins, and thus we cannot extrapolate that increasing $\mathrm{BMI}$ is associated with lower $\mathrm{EBC}$ nitrate and increased alternative NOS pathways.

Our study strictly excluded patients with any respiratory diagnosed disease or symptoms, smoking history or chronic oxygen therapy, or impairment of the immune system, as well as moderate or severe obesity [31]. The 21 patients included in this analysis showed similar distribution of age, gender or comorbidities except the greater incidence of pre-diagnosed hypertension in the obese group [5,33]. Despite the 
Citation: Fernandez-Bustamante A, Seres T, Agazio A, Pennington AT, Christians U, et al. (2015) Exhaled Breath Condensate Nitrate Levels are Inversely Associated with the Body Mass Index of Patients without Respiratory Disease. J Pulm Respir Med 5: 243. doi: 10.4172/2161-105X.1000243

Page 7 of 8

reduced sample, we are confident that our patients are representative of otherwise healthy obese individuals. The generalization of our findings, however, may be limited by the fact that we analyzed metabolites with relatively wide variability in a small number of patient samples. Analysis of inflammatory or oxidative biomarkers in EBC samples is attractive because of its non-invasiveness and safety. However, its widespread use is limited by the difficulty in detecting diluted biomarkers, lack of information about their source within the airway anatomy, and lack of standardization of collection and data analysis [57,58]. We cannot exclude an effect of individual diets [59,60], exercise habits, lipid profiles or undiagnosed hyperglycemia or other conditions. However, tracheally collected EBC nitrate levels are less affected by a dietary nitrate load than oropharyngeal nitrate concentrations [60] and also avoids contamination with saliva and the influence that salivary bacteria has on NO metabolites [59,60]. Preoperative fasting or anesthesia medications were similar in all patients but have an unknown effect on EBC or plasma metabolites.

\section{Conclusions}

We found an inverse correlation of BMI with concentrations of nitrate in the EBC from surgical patients without respiratory or immune conditions. Patients who were obese (defined in our study as BMI 25-34.9) had significantly lower EBC nitrate levels than patients with normal weights $(\mathrm{BMI}<25)$. Our observation of lower nitrate in the lung of obese patients needs to be further explored. If confirmed, this apparent obesity-related dampening of lung nitrosative stress could provide a mechanistic insight into the unexplained findings underlying the obesity-ARDS paradox.

\section{Acknowledgements}

The authors thank Allison J. Janocha, BSE and Serpil C. Erzurum, MD, from the Department of Pathobiology and Lerner Research Institute (NC22), the Cleveland Clinic Foundation and Case Western Reserve University School of Medicine (Cleveland, $\mathrm{OH}, \mathrm{USA}$ ) for their technical assistance with nitrite and nitrate measurements (funded by HL103453 and HL081064).

\section{Funding}

AFB - University of Colorado School of Medicine Department of Anesthesiology (Aurora, CO, USA) Seed Grant, 2012 Foundation for Anesthesia Education \& Research Clinical/Translational-Mentored Research Training Grant.

Rest - institutional support from University of Colorado SOM (Aurora, CO, USA) Departments of Anesthesiology (TS, AA, ATP, UC and JK) and Medicine (JER)

\section{References}

1. Lakka HM, Laaksonen DE, Lakka TA, Niskanen LK, Kumpusalo E, et al. (2002) The metabolic syndrome and total and cardiovascular disease mortality in middle-aged men. JAMA 288: 2709-2716.

2. Olson AL, Zwillich C (2005) The obesity hypoventilation syndrome. Am J Med 118: 948-956.

3. Guenette JA, Jensen D, O'Donnell DE (2010) Respiratory function and the obesity paradox. Curr Opin Clin Nutr Metab Care 13: 618-624.

4. Lam JC, Mak JC, Ip MS (2012) Obesity, obstructive sleep apnoea and metabolic syndrome. Respirology 17: 223-236.

5. Flegal KM, Kit BK, Orpana H, Graubard BI (2013) Association of all-cause mortality with overweight and obesity using standard body mass index categories: a systematic review and meta-analysis. JAMA 309: 71-82.

6. Lavie CJ, Milani RV, Ventura HO (2009) Obesity and cardiovascular disease: risk factor, paradox, and impact of weight loss. J Am Coll Cardiol 53: 19251932.

7. Fonarow GC, Srikanthan P, Costanzo MR, Cintron GB, Lopatin M; ADHERE Scientific Advisory Committee and Investigators (2007) An obesity paradox in acute heart failure: analysis of body mass index and inhospital mortality for 108,927 patients in the Acute Decompensated Heart Failure National Registry. Am Heart J 153: 74-81.
8. Mullen JT, Moorman DW, Davenport DL (2009) The obesity paradox: body mass index and outcomes in patients undergoing nonbariatric general surgery. Ann Surg 250: 166-172.

9. Florez H, Castillo-Florez S (2012) Beyond the obesity paradox in diabetes: fitness, fatness, and mortality. JAMA 308: 619-620.

10. Andersen KK, Olsen TS (2013) The obesity paradox in stroke: Lower mortality and lower risk of readmission for recurrent stroke in obese stroke patients. International journal of stroke: official journal of the International Stroke Society.

11. Lavie CJ, McAuley PA, Church TS, Milani RV, Blair SN (2014) Obesity and cardiovascular diseases: implications regarding fitness, fatness, and severity in the obesity paradox. J Am Coll Cardiol 63: 1345-54.

12. Memtsoudis SG, Bombardieri AM, Ma Y, Walz JM, Chiu YL, et al. (2012) Mortality of patients with respiratory insufficiency and adult respiratory distress syndrome after surgery: the obesity paradox. J Intensive Care Med 27: 306311

13. Morris AE, Stapleton RD, Rubenfeld GD, Hudson LD, Caldwell E, et al. (2007) The association between body mass index and clinical outcomes in acute lung injury. Chest 131: 342-348.

14. O’Brien JM Jr, Phillips GS, Ali NA, Lucarelli M, Marsh CB, et al. (2006) Body mass index is independently associated with hospital mortality in mechanically ventilated adults with acute lung injury. Crit Care Med 34: 738-744.

15. Gong MN, Bajwa EK, Thompson BT, Christiani DC (2010) Body mass index is associated with the development of acute respiratory distress syndrome. Thorax 65: 44-50.

16. Anzueto A, Frutos-Vivar F, Esteban A, Bensalami N, Marks D, et al. (2011) Influence of body mass index on outcome of the mechanically ventilated patients. Thorax 66: 66-73.

17. Stapleton RD, Dixon AE, Parsons PE, Ware LB, Suratt BT; NHLBI Acute Respiratory Distress Syndrome Network (2010) The association between BMI and plasma cytokine levels in patients with acute lung injury. Chest 138: 568577

18. Fernandez-Bustamante A, Repine JE (2013) Adipose-Lung Cell Crosstalk in the Obesity-ARDS Paradox. J Pulmon Resp Med 3: 1-7.

19. Fernandez-Bustamante A, Repine JE (2012) The Obesity ARDS Paradox: "A Pre-Conditioning Cloud". J Pulmon Resp Med 2: e122.

20. Toda N, Okamura T (2013) Obesity impairs vasodilatation and blood flow increase mediated by endothelial nitric oxide: an overview. J Clin Pharmacol 53: 1228-1239

21. Stühlinger MC, Tsao PS, Her JH, Kimoto M, Balint RF, et al. (2001) Homocysteine impairs the nitric oxide synthase pathway: role of asymmetric dimethylarginine. Circulation 104: 2569-2575.

22. Holguin F, Comhair SA, Hazen SL, Powers RW, Khatri SS, et al. (2013) An association between $\mathrm{L}$-arginine/asymmetric dimethyl arginine balance, obesity and the age of asthma onset phenotype. Am J Respir Crit Care Med 187: 153159

23. Sutherland ER, Goleva E, King TS, Lehman E, Stevens AD, et al. (2012) Cluster analysis of obesity and asthma phenotypes. PLoS One 7: e36631.

24. Lombardi C, Gargioni S, Gardinazzi A, Canonica GW, Passalacqua G (2011) Impact of bariatric surgery on pulmonary function and nitric oxide in asthmatic and non-asthmatic obese patients. J Asthma 48: 553-557.

25. Kim SH, Kim TH, Lee JS, Koo TY, Lee CB, et al. (2011) Adiposity, adipokines and exhaled nitric oxide in healthy adults without asthma. J Asthma 48: 177182.

26. Holguin F, Rojas M, Brown LA, Fitzpatrick AM (2011) Airway and plasma leptin and adiponectin in lean and obese asthmatics and controls. J Asthma 48: 217223.

27. D'Alessio FR, Tsushima K, Aggarwal NR, Mock JR, Eto Y, et al. (2012) Resolution of experimental lung injury by monocyte-derived inducible nitric oxide synthase. J Immunol 189: 2234-2245.

28. Pastva AM, Walker JK, Maddox LA, Mukherjee S, Giamberardino C, et al. (2011) Nitric oxide mediates relative airway hyporesponsiveness to lipopolysaccharide in surfactant protein A-deficient mice. Am J Respir Cell Mol Biol 44: 175-184.

29. Sittipunt C, Steinberg KP, Ruzinski JT, Myles C, Zhu S, et al. (2001) Nitric oxide and nitrotyrosine in the lungs of patients with acute respiratory distress syndrome. Am J Respir Crit Care Med 163: 503-510. 
Citation: Fernandez-Bustamante A, Seres T, Agazio A, Pennington AT, Christians U, et al. (2015) Exhaled Breath Condensate Nitrate Levels are Inversely Associated with the Body Mass Index of Patients without Respiratory Disease. J Pulm Respir Med 5: 243. doi: 10.4172/2161-105X.1000243

Page 8 of 8

30. Lundberg JO, Weitzberg E, Gladwin MT (2008) The nitrate-nitrite-nitric oxide pathway in physiology and therapeutics. Nat Rev Drug Discov 7: 156-167.

31. Fernandez-Bustamante A, Klawitter J, Repine JE, Agazio A, Janocha AJ, et al. (2014) Early effect of tidal volume on lung injury biomarkers in surgical patients with healthy lungs. Anesthesiology 121: 469-481.

32. ARDSNet (2000) Ventilation with lower tidal volumes as compared with traditional tidal volumes for acute lung injury and the acute respiratory distress syndrome. The Acute Respiratory Distress Syndrome Network. N Engl J Med 342: $1301-1308$

33. CDC/NCHS. Defining Overweight and Obesity.

34. Erzurum SC, Ghosh S, Janocha AJ, Xu W, Bauer S, et al. (2007) Higher blood flow and circulating NO products offset high-altitude hypoxia among Tibetans. Proc Natl Acad Sci U S A 104: 17593-17598.

35. Rihák V, Zatloukal P, Chládková J, Zimulová A, Havlínová Z, et al. (2010) Nitrite in exhaled breath condensate as a marker of nitrossative stress in the airways of patients with asthma, COPD, and idiopathic pulmonary fibrosis. $J$ Clin Lab Anal 24: 317-322.

36. Araneda OF, Guevara AJ, Contreras C, Lagos N, Berral FJ (2012) Exhaled breath condensate analysis after long distance races. Int J Sports Med 33: 955-961.

37. Arcêncio L, Vento DA, Bassetto S, Evora PR, Rodrigues AJ (2013) Exhaled nitrite/nitrate levels as a marker of respiratory complications after heart valve surgery. J Crit Care 28: 533.

38. Pheng LH, Francoeur C, Denis M (1995) The involvement of nitric oxide in a mouse model of adult respiratory distress syndrome. Inflammation 19: 599-610.

39. Haddad IY, Pataki G, Hu P, Galliani C, Beckman JS, et al. (1994) Quantitation of nitrotyrosine levels in lung sections of patients and animals with acute lung injury. J Clin Invest 94: 2407-2413.

40. Enkhbaatar P, Murakami K, Shimoda K, Mizutani A, Traber L, et al. (2003) The inducible nitric oxide synthase inhibitor BBS-2 prevents acute lung injury in sheep after burn and smoke inhalation injury. Am J Respir Crit Care Med 167: 1021-1026.

41. Gole MD, Souza JM, Choi I, Hertkorn C, Malcolm S, et al. (2000) Plasma proteins modified by tyrosine nitration in acute respiratory distress syndrome. Am J Physiol Lung Cell Mol Physiol 278: L961-967.

42. Lamb NJ, Gutteridge JM, Baker C, Evans TW, Quinlan GJ (1999) Oxidative damage to proteins of bronchoalveolar lavage fluid in patients with acute respiratory distress syndrome: evidence for neutrophil-mediated hydroxylation, nitration, and chlorination. Crit Care Med 27: 1738-1744.

43. Ware LB, Magarik JA, Wickersham N (2013) Low plasma citrulline levels are associated with acute respiratory distress syndrome in patients with severe sepsis. Crit Care 17: R10.

44. Vemmos K, Ntaios G, Spengos K, Savvari P, Vemmou A, et al. (2011) Association between obesity and mortality after acute first-ever stroke: the obesity-stroke paradox. Stroke 42: 30-36.

45. Sakr Y, Elia C, Mascia L, Barberis B, Cardellino S, et al. (2012) Being overweight or obese is associated with decreased mortality in critically ill patients: a retrospective analysis of a large regional Italian multicenter cohort. J Crit Care 27: 714-721.
46. King P, Mortensen EM, Bollinger M, Restrepo MI, Copeland LA, et al. (2013) Impact of obesity on outcomes for patients hospitalised with pneumonia. Eur Respir J 41: 929-934

47. Nafiu OO, Kheterpal S, Moulding R, Picton P, Tremper KK, et al. (2011) The association of body mass index to postoperative outcomes in elderly vascula surgery patients: a reverse J-curve phenomenon. Anesth Analg 112: 23-29.

48. Durward CM, Hartman TJ, Nickols-Richardson SM (2012) All-cause mortality risk of metabolically healthy obese individuals in NHANES III. J Obes 2012 460321

49. Barry VW, Baruth M, Beets MW, Durstine JL, Liu J, et al. (2014) Fitness vs. fatness on all-cause mortality: a meta-analysis. Prog Cardiovasc Dis 56: 382 390

50. Davids M, Swieringa E, Palm F, Smith DE, Smulders YM, et al. (2012) Simultaneous determination of asymmetric and symmetric dimethylarginine, L-monomethylarginine, L-arginine, and L-homoarginine in biological samples using stable isotope dilution liquid chromatography tandem mass spectrometry. J Chromatogr B Analyt Technol Biomed Life Sci 900: 38-47.

51. Di Gangi IM, Pirillo P, Carraro S, Gucciardi A, Naturale M, et al. (2012) Online trapping and enrichment ultra performance liquid chromatography-tandem mass spectrometry method for sensitive measurement of "arginine-asymmetric dimethylarginine cycle" biomarkers in human exhaled breath condensate. Anal Chim Acta 754: 67-74

52. Carraro S, Giordano G, Piacentini G, Kantar A, Moser S, et al. (2013) Asymmetric dimethylarginine in exhaled breath condensate and serum of children with asthma. Chest 144: 405-410.

53. Lüneburg N, Harbaum L2, Hennigs JK3 (2014) The endothelial ADMA/NO pathway in hypoxia-related chronic respiratory diseases. Biomed Res Int 2014 501612

54. Zakrzewicz D, Eickelberg O (2009) From arginine methylation to ADMA: a novel mechanism with therapeutic potential in chronic lung diseases. BMC Pulm Med 9: 5.

55. Lakshmy A, Jean H, Farook J, Christina K (214) The Relationship Of Arginine And Its Metabolites In Exhaled Breath Condensate To Lung Function: American Thoracic Society.

56. Sansbury BE, Cummins TD, Tang Y, Hellmann J, Holden CR, et al. (2012) Overexpression of endothelial nitric oxide synthase prevents diet-induced obesity and regulates adipocyte phenotype. Circ Res 111: 1176-1189.

57. Silkoff PE, Erzurum SC, Lundberg JO, George SC, Marczin N, et al. (2006) ATS workshop proceedings: exhaled nitric oxide and nitric oxide oxidative metabolism in exhaled breath condensate. Proc Am Thorac Soc 3: 131-145.

58. Carter SR, Davis CS, Kovacs EJ (2012) Exhaled breath condensate collection in the mechanically ventilated patient. Respir Med 106: 601-613.

59. Malinovschi A, Janson C, Holm L, Nordvall L, Alving K (2009) Basal and induced $\mathrm{NO}$ formation in the pharyngo-oral tract influences estimates of alveolar NO levels. J Appl Physiol (1985) 106: 513-519.

60. Marteus H, Törnberg DC, Weitzberg E, Schedin U, Alving K (2005) Origin of nitrite and nitrate in nasal and exhaled breath condensate and relation to nitric oxide formation. Thorax 60: 219-225. 